\title{
Hollow-Cone Foucault Imaging Method for Magnetic Structure Observations
}

\author{
K. Harada ${ }^{1,2}$, A. Kawaguchi², A. Kotani², Y. Fujibayashi², K. Shimada ${ }^{1}$, and S. Mori ${ }^{2}$ \\ 1. CEMS, RIKEN (The Institute of Physical and Chemical Research), Hatoyama, Saitama 350-0395, Japan \\ 2. Department of Materials Science, Osaka Prefecture University, Sakai, Osaka 599-8531, Japan
}

In conventional transmission electron microscopy (TEM), observation of magnetization structures and dielectric polarization structures are difficult to observe because these structures weakly interact with electron beams. Lorentz microscopy was developed to observe these structures effectively and practically. In Lorentz microscopy, however, Fresnel and Foucault imaging methods have the following disadvantages: in the Fresnel imaging method no additional contrast can be generated on the domains because of just defocusing for images and in the Foucault imaging method only the filtered out information can be obtained from selected domains. To overcome these difficulties, hollow-cone Foucault (HCF) imaging method was developed [1], where an incident electron beam on the specimen was tilted with respect to the optical axis and was circulated in all azimuths around the optical axis. Both magnetic domains and domain walls were simultaneously visualized with sufficient contrasts under the infocus condition. Furthermore, it was confirmed that schlieren imaging method [2], known as a highspeed imaging method applicable to low refractive index media, was realized.

Figure 1 shows a schematic diagram of the optical system for the HCF imaging. The parallel electron beams having less than $10^{-6}$ rad diffusion angle are irradiated on the specimen with the inclination angles in $\mathrm{X}$ and $\mathrm{Y}$ directions controlled by using the beam deflector system placed above the specimen. The circulating electron beam is illuminated in all azimuthal directions around the optical axis. This special condition leads to realization of small-angle hollow-cone beams with an inclination angle as small as $10^{-4} \mathrm{rad}$. The experiment was performed using a 200-kV thermal field-emission TEM (JEM-2100F) and the HCF images were recorded with a $2 \mathrm{k} \times 2 \mathrm{k}$ pixel charge-coupled device camera (Ultrascan camera). Each image was recorded through 12 turns in the illumination azimuthal rotation in 7.5 seconds, corresponding to $1.6 \mathrm{~Hz}$. An $\mathrm{Fe}_{0.88} \mathrm{Ga}_{0.12}$ alloy, which has large magnetostriction at room temperature, was thinned to $250 \mathrm{~nm}$ thickness by focused ion beam instrument (NB-5000).

Figures 2(a) and (b) show the bright-field and dark-field HCF images for different inclination angle conditions. The selected area aperture with opening size of $100 \mu \mathrm{m}$ in diameter corresponding to the $1.30 \times 10^{-3}$ rad was utilized as the angle-limiting tool. Figure 2(a) is a bright-field HCF image, where domain walls are observed as white lines and several domains have slight dark contrast. Figure 2(b) is a dark-field HCF image, where domain walls are observed with black lines and several domains also have slight bright contrast. The contrast in the dark-field HCF image in Fig. 2(b) is reversed with respect to the bright-field HCF image in Fig. 2(a).

In addition, schlieren imaging mode, obtained under specific inclination angle of the illumination beam between the bright-field and dark-field modes, can qualitatively show magnetic field leaking from the specimen over a wide range and to a far distance.

The developed HCF imaging method has advantages of both Fresnel and Foucault imaging methods: therefore, HCF method can be called the third Lorentz microscopy. We hope the HCF method will be widely used for analyzing electromagnetic properties in materials in the future. 
References:

[1] K. Harada et al, Appl. Phys. Exp., 12 (2019), https://doi.org/10.7567/1882-0786/ab0523

[2] H. Water, in "Handbuch der Physik”, 24, 555 (Springer, Germany 1956).

[3] Authors would like to thank Dr. Y. A. Ono of RIKEN, CEMS for his valuable discussion, Prof. H. Numakura at Osaka Prefecture University for providing us the $\mathrm{Fe}_{0.88} \mathrm{Ga}_{0.12}$ alloy, and $\mathrm{Mr}$. K. Uchihashi of Osaka Prefecture University for his technical support.

[4] This work was supported by KAKENHI, Grant-in-Aid for Scientific Research ((B) 18H03475).

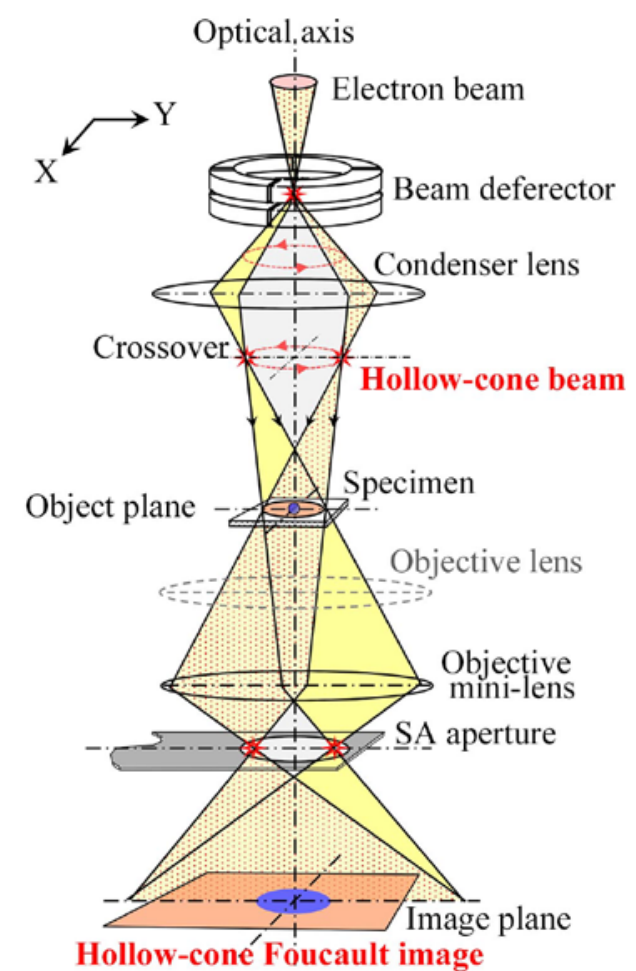

Hollow-coné Foucault image
Figure 1. Schematic diagram of the optical system for the HCF imaging. Instead of a conventional objective lens, an objective mini-lens is used to focus the crossover on the selected area (SA) aperture plane. The SA aperture works as an angle limiting tool just like the conventional objective aperture. The beam deflectors above the condenser lens incline and circulate the illumination beam in all azimuthal direction around the optical axis.
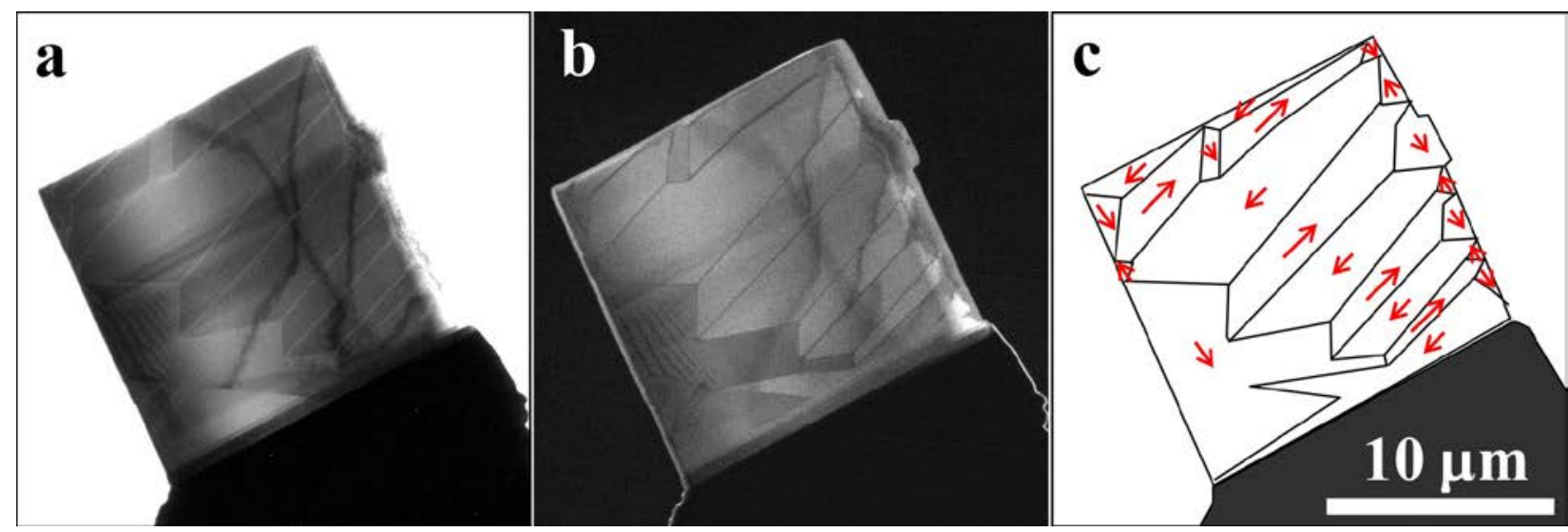

Figure 2. (a) Bright-field HCF image for $2.8 \times 10^{-4}$ rad inclination angle, (b) dark-field HCF image for $4.4 \times 10^{-4}$ rad inclination angle, (c) schematic drawing of magnetic domain structure. The bright and darkfield HCF images can simultaneously visualize both magnetic domains and magnetic domain walls under the infocus condition. 